\title{
Measurement of Partial Conductivity of 8YSZ by Hebb-Wagner Polarization Method
}

\author{
Dae-Kwang Lim, Jae-Geun Guk, Hyen-Seok Choi, and Sun-Ju Song ${ }^{\dagger}$ \\ Ionics Lab, School of Materials Science and Engineering Chonnam National University, Gwang-Ju 61186, Korea
}

(Received May 12, 2015; Revised May 28, 2015; Accepted June 1, 2015)

\begin{abstract}
The electrolyte is an important component in determining the performance of Fuel Cells. Especially, investigation of the conduction properties of electrolytes plays a key role in determining the performance of the electrolyte. The electrochemical properties of Yttrium stabilized zirconia (YSZ) were measured to allow the use of this material as an electrolyte for solid oxide fuel cells (SOFC) in the temperature range of $700-1000^{\circ} \mathrm{C}$ and in $0.21 \leq p \mathrm{O}_{2} / \mathrm{atm} \leq 10^{-23}$. A Hebb-Wagner polarization experimental cell was optimally manufactured; here we discuss typical problems associated with making cells. The partial conductivities due to electrons and holes for 8YSZ, which is known as a superior oxygen conductor, were obtained using I-V characteristics based on the Hebb-Wagner polarization method. Activation energies for holes and electrons are $3.99 \pm 0.17 \mathrm{eV}$ and $1.70 \pm 0.06 \mathrm{eV}$ respectively. Further, we calculated the oxygen ion conductivity with electron, hole, and total conductivity, which was obtained by DC four probe conductivity measurements. The oxygen ion conductivity was dependent on the temperature; the activation energy was $0.80 \pm 0.10 \mathrm{eV}$. The electrolyte domain was determined from the top limit, bottom limit, and boundary $(\mathrm{p}=\mathrm{n})$ of the oxygen partial pressure. As a result, the electrolyte domain was widely presented in an extensive range of oxygen partial pressures and temperatures.
\end{abstract}

Key words : Yttrium stabilized zirconia, Hebb-Wagner ion-blocking method, Electrolytic domain, Partial electronic conductivity

\section{Introduction}

$\mathbf{F}$ uel cell displays an excellent efficiency during chemical to electrical energy transformation. Since the conversion is direct without any involvement of combustion, no pollutant such as SOx or NOx is emitted, which makes the process highly popular in the green energy sector. ${ }^{1)}$ Solid oxide fuel cell (SOFC), especially, exhibits the highest transform efficiency up to the temperature range of $600-1000^{\circ} \mathrm{C}$, and thus can perform accelerated reaction without any presence of noble catalyst. ${ }^{2)}$ Furthermore, the process becomes more viable since the waste heat at high temperatures can be recycled, and internal reforming reaction is feasible. It also can adopt a variety of fuels such as LNG, LPG, propane gas, bio fuels, etc.

Yttria-stabilized zirconia (YSZ) has been used as a typical electrolyte for SOFC featuring high phase stability and high ionic conductivity at the general operation range of fuel cell. Thus, it has been adopted as a reference material for the new development of an electrolyte and its property evaluation. YSZ, however, has a shortcoming of drastic decrease in ionic conductivity at lower temperatures. This has stimulated many studies to overcome the weakness. However, no

Corresponding author : Sun-Ju Song

E-mail : song@chonnam.ac.kr

Tel : +82-62-530-1706 Fax : +82-62-530-1699 new electrolyte material is good enough to replace YSZ. ${ }^{4,5)}$

Electrolyte is required to have high ionic and low electric conductivities. Electric conductivity is normally insensitive to chemical potential, and maintains the conductive characteristics at the presence of chemical potential gradient. However, determining its electrolytic domain by conductivity measurement is an important subject for the operation of a solid electrolyte fuel cell. YSZ, an excellent ionic conductor, shows a very low electric conductivity, and thus, researchers have paid less attention on that issue. Note, however, that electric conduction can limit the cell efficiency by reducing the driving force.

In this study, we measured electric conductivity by HebbWagner polarization method. The method uses an ion-blocking electrode, and measures the genuine electric conduction quantitatively by generating chemical potential difference large enough to offset the applied external voltage difference to drive transport. ${ }^{6,7)}$ The setup and measurement are relatively simple in principle, but require delicate precautions in actual practice. And we provided the detailed procedure to overcome the difficulties. We thus measured the current-voltage characteristic of YSZ electrolyte and analyzed its physical meaning.

\section{Experimental Procedure}

A commercial powder (8YSZ, Totho) with an average particle size of $40 \mathrm{~nm}$ was formed into a disc in CIP under 150 
$\mathrm{MPa}$, and sintered at $1450^{\circ} \mathrm{C}$ for $10 \mathrm{~h}$ in air. We cut the sintered polycrystalline $8 \mathrm{YSZ}$ specimen into a $1.5 \mathrm{~mm}$ thick disc for Hebb-Wagner polarization experiment. We also cut $1.8 \times 1.8 \times 11 \mathrm{~mm}$ samples from the specimen and polished their surfaces to roughness less than $1 \mu \mathrm{m}$ for conductivity measurement. Fig. 1 shows the X-ray diffraction pattern of $8 Y S Z$, scanned at $2 \%$ min by XRD (D/MAX Ultima III, Rigaku, Japan) and analyzed the pattern by profile matching.

Figure 2 is the image of fractured 8YSZ by scanning electron microscopy (SEM, SS-550, Shimadzu, Japan), which indicates a very dense nature of the specimen. Its bulk density measured by the Archimedes method was higher than $97.5 \%$.

We measured electric conductivity of samples by the 4probe method, combining a digital multimeter (Keithley 2700) and a current source (Keithley 6220) at various temperatures $\left(700 \leq\right.$ Temp. $\left.\left({ }^{\circ} \mathrm{C}\right) \leq 1000\right)$ and partial pressures of oxygen $\left(0.21 \leq p \mathrm{O}_{2}(\mathrm{~atm}.) \leq 10^{-23}\right)$. The partial conductivity of 8YSZ was measured by preparing a Hebb-Wagner polarization cell with an ion-blocking electrode (Pt foil) on one side and a reversible electrode ( $\mathrm{Pt}$ mesh) on the other.

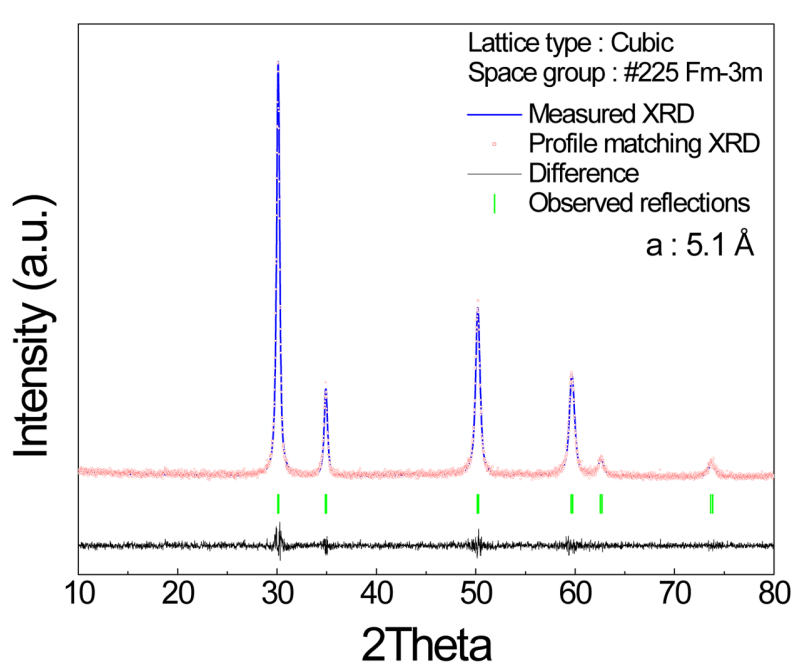

Fig. 1. Room-temperature XRD pattern of the 8YSZ.

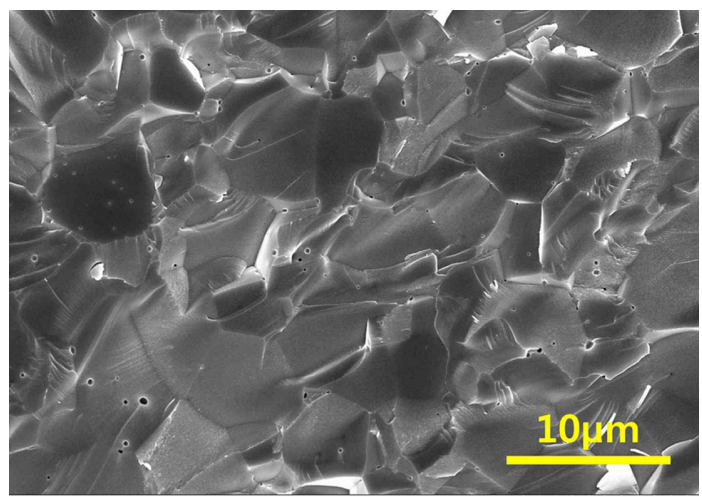

Fig. 2. SEM image of the fractured $8 \mathrm{YSZ}$ sintered at $1450^{\circ} \mathrm{C}$ in air.

\section{Results and Discussion}

\subsection{Configuration of Hebb-Wagner polarization cell}

In the frame of the Hebb-Wagner polarization scheme, many cell models and test methods are available for electrochemical analysis since the method introduced by Hebb, separating ion and electron conductivities for $\mathrm{Ag}_{2} \mathrm{~S}{ }^{6}{ }^{6}$ In this study, we first adopted a cell configuration which has much less contact area compared to other micro-contact cells to establish the steady-state of cell faster. Fig. 3 shows image of the cell and its schematic with small and clearly-defined contact area, which can make the response during measurement fast. However, leakage through the area other than that of the electrode was problematic. The leakage caused unwanted interaction between oxygen ion and the surrounding air, which led the conclusion that the cell was not appropriate to produce accurate data.

We thus adopted the four-probe setup as shown in Fig. 4, to eliminate the possibility of underestimation of electric conductivity, which often occur with two electrodes due to over-voltage at the electrodes. Mass and charge transport through the area other than that of the electrode was completely blocked off by double sealing.

Figure 5 shows a typical cell which was problematic during trials. Fig. 5(a) is two incidents that occurred when the specimen was not properly fixed.

The two electrodes, ion-blocking electrode on one side and reversible electrode on the other, are normally fixed with application of some load or of high-temperature ceramic bond. If they are not properly secured, gaps can be formed between either electrode and sample, and sealing material can penetrate into the cell. In addition, pressure build-up by polarization during measurement also can open a gap. These incidents happen on the blocking electrode side, only

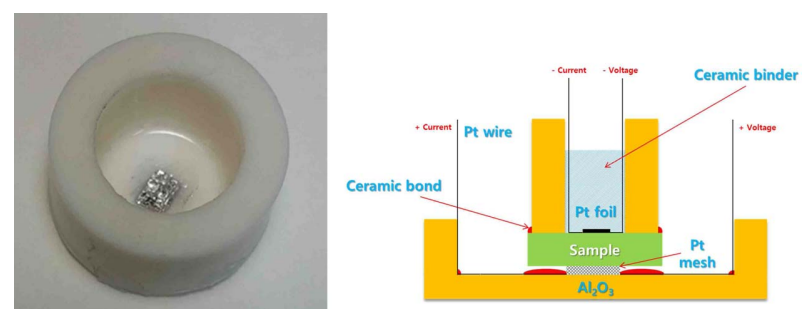

Fig. 3. Schematic diagram of the Hebb-Wagner polarization cell of micro-contact type.

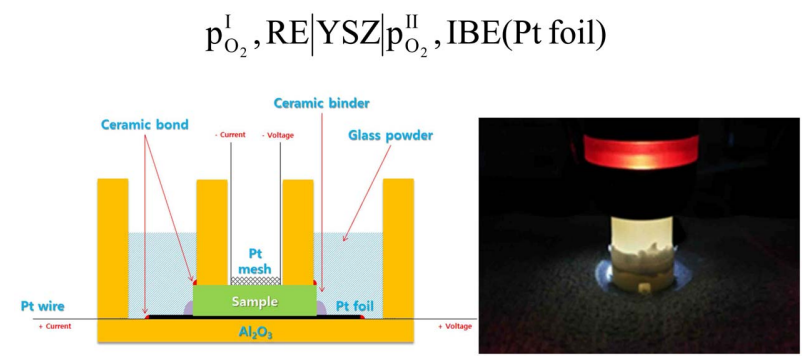

Fig. 4. Schematic and image of the Hebb-Wagner polarization cell in a four-probe setup. 


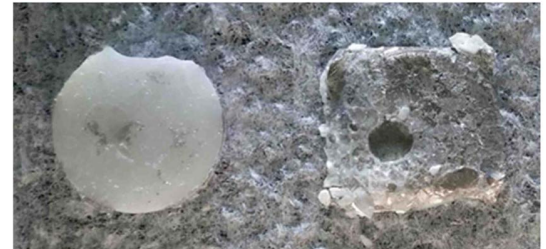

(a)

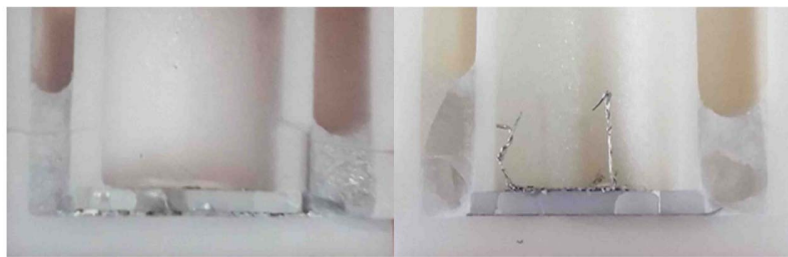

(b)

Fig. 5. (a) Problems with the unfixed Hebb-Wagner polarization cells, (b) Problems with the structurally-defective Hebb-Wagner polarization cells.

when oxygen migrates toward the blocking electrode.

Figure 5(b) shows problems occurred due to lack of structural integrity of cell during sealing procedure. The sealing material, Pyrex glass, seals the cell when it is melted down and pores are removed by moving upward. If, however, the alumina guide, that prevents glass infiltration into the reversible electrode, is bigger than the sample, pores will have a tendency to remain inside. Thus, it is important to make the cell configuration such that pore can easily move upward. After fixing the components with ceramic binder and bond as shown in Fig. 5, the side of cell from the reversible electrode to blocking electrode should be sealed from air with a good sealing material to have reliable data production.

\subsection{Partial conductivity from Hebb-Wagner polarization}

In YSZ, the defect reaction with yttria proceeds by the following equation expressed in Kroger and Vink notation:

$$
Y_{2} O_{3} \rightarrow 2 Y_{Z r}^{\prime}+3 O_{O}^{\times}+V_{O}^{\bullet}
$$

Here, $Y_{Z r}^{\prime}$ represents for yttrium on the zirconium site, $O_{O}^{\times}$for neutral oxygen on its own site, and $V_{O}^{\cdot \bullet}$ for vacancy on the oxygen site, respectively. Assuming that anti-Frankel defect is the domination defect in a stoichiometric YSZ.

$$
\begin{aligned}
& \text { 1) Internal equilibria } \\
& K_{I}=n \cdot p \\
& K_{A F}=\left[O_{i}^{\prime \prime}\right]\left[V_{O}^{\bullet \cdot}\right]
\end{aligned}
$$

Where $\mathrm{K}, \mathrm{n}$, and $\mathrm{p}$ are the equilibrium constants, concentrations of electron and hole, respectively.

$$
\begin{aligned}
& \text { 2) External equilibria } \\
& K_{\mathrm{Re}}=n^{2}\left[V_{O}^{\ddot{*}}\right] P_{O_{2}}^{1 / 2} \\
& K_{O x}=p^{2}\left[O_{i}^{\prime /}\right] P_{O_{2}}^{-1 / 2}
\end{aligned}
$$

Finally, the electric neutrality condition gives:

$$
n+2\left[O_{i}^{\prime \prime}\right]+\left[O_{Z r}^{\prime}\right]=p+2\left[V_{O}^{\bullet}\right]
$$

Here, [j] is concentrations of point defect, j. Based on the above equation, a defect diagram for YSZ is constructed as shown in Fig. 6.

Here, $\left[O_{i}^{\prime \prime}\right]$ is the concentration of interstitial oxygen ion. The $8 \mathrm{~mol} \%$-doped YSZ prepared for this study can be considered as a high-doped case, and its characteristics in the electrolytic domain can be represented as $2\left[V_{O}^{\ddot{ }}\right]=\left[Y_{Z r}^{\prime}\right]$. Therefore, conductivities by electron and hole can be expressed in terms of oxygen partial pressure as:

$$
\begin{aligned}
& \sigma_{n} \propto P_{O_{2}}^{-1 / 4} \\
& \sigma_{p} \propto P_{O_{2}}^{+1 / 4}
\end{aligned}
$$

Conductivity can also be expressed in terms of carrier concentration ([]), charge (q), and mobility (u):

$$
\sigma_{j}=[]_{j} \cdot q \cdot u_{j}
$$

At the ion-blocking electrode ( $\mathrm{Pt}$ foil) of cell, oxygen inside of the specimen meets electron driven by external current (or voltage) as:

$$
\mathrm{O}_{2}+4 e^{-} \leftrightarrow 2 O^{2-}
$$

In terms of chemical and electro-chemical potential differences, the above equation can be rewritten as:

$$
\mu_{O_{2}}^{\prime \prime}-\mu_{O_{2}}^{\prime \prime}+4\left(\eta_{e}^{\prime \prime}-\eta_{e}^{\prime}\right)=2\left(\eta_{O^{2-}}^{\prime \prime}-\eta_{O^{2-}}^{\prime}\right)
$$

Under the applied constant current between reversible and ion-blocking electrodes, both ion (oxygen) and electron migrate initially. However, once oxygen flow is blocked by the ion-blocking electrode, the flow decreases, and oxygen partial pressure within the electrode also decreases. This creates difference in oxygen partial pressure between both ends of sample, which leads to a concentration gradient. Oxygen thus flows from the reversible electrode to the ion-

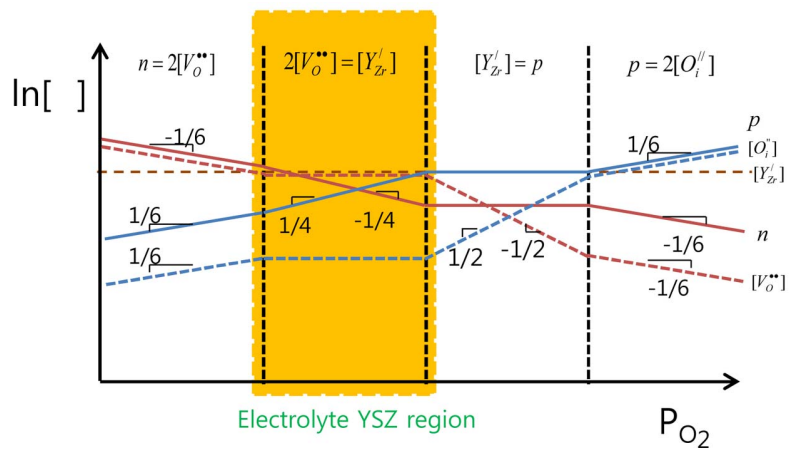

Fig. 6. Defect diagram with the concentrations of carriers vs. $p_{\mathrm{O} 2}$. 
blocking electrode. Eventually, oxygen gradients by constant current and from the reversible electrode will be equalized, and a steady-state will be established as the oxygen ion flow becomes zero as:

$$
J_{O^{2-}}=-\frac{\sigma_{O^{2-}}}{4 F} \nabla \eta_{O^{2-}}=0
$$

Thus, by measuring voltage of both ends of sample at steady-state, oxygen chemical potential difference can be estimated. Fig. 7 shows the profile of current-voltage characteristic of the 8YSZ, reaching to the steady-state. Since current density at steady-state is due to only electron, the slope of the current-voltage curve can provide partial electric conductivity according to the following equation:

$$
\sigma_{e}\left(\mu_{O_{2}}=\mu_{O_{2}}^{\prime \prime}\right)=-\frac{L}{A}\left(\frac{d j_{e}}{d U}\right)
$$

Here, L, A, and U represent length, area, and voltage at both ends of electrodes in the sample, respectively.

It is apparent that the curves of OCV state at the applied constant current consist of a drastic increase in voltage, followed by convergence to the steady-state. It is also confirmed that, if applied constant current is shut off, the curves go back to the original state with no voltage difference.

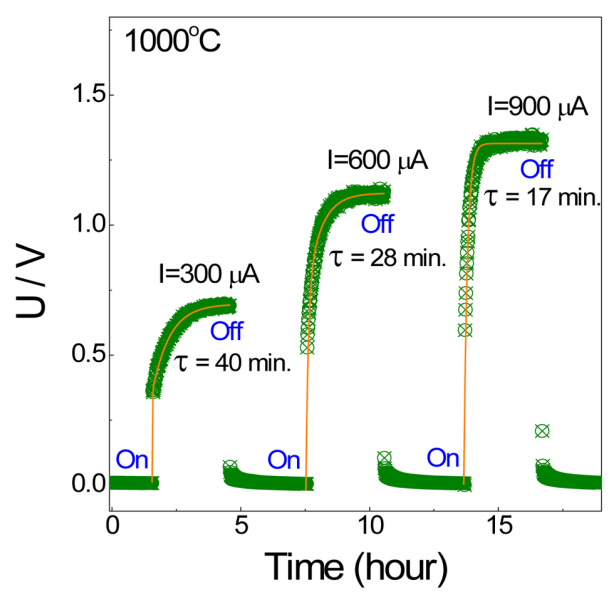

Fig. 7. Current-voltage characteristic of the 8YSZ.

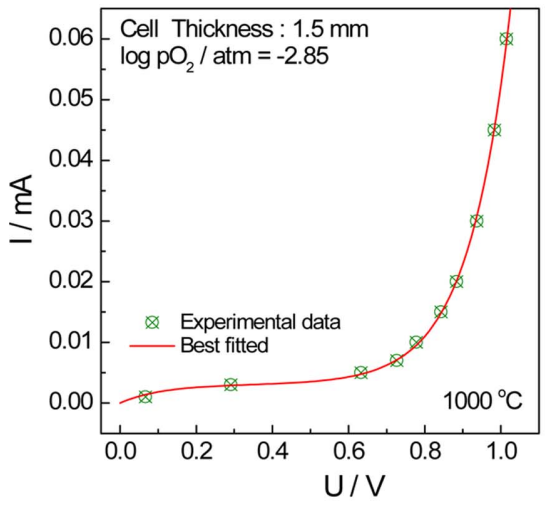

Fig. 8. Partial conductivities of the 8YSZ.
Electric conductivity is composed of contributions from electron and hole:

$$
\sigma_{e}=\sigma_{n}+\sigma_{p}
$$

In case of YSZ at the given partial pressures of oxygen (0.21-10 ${ }^{-23} \mathrm{~atm}$.), conductivities by electron and hole are proportional to the partial pressure of oxygen to the powers of $1 / 4$ and $1 / 4$, respectively:

$$
\sigma_{e}=\sigma_{n}^{*}\left(\frac{p O_{2}}{p O_{2}}\right)^{-1 / 4}+\sigma_{p}^{*}\left(\frac{p O_{2}}{p O_{2}{ }^{*}}\right)^{1 / 4}
$$

Therefore, considering the chemical potential of oxygen molecule and the partial pressure of oxygen, the partial conductivities of electron and hole under the applied constant current and voltage at steady-state is:

$$
I_{e}=\frac{A R T}{L F}\left[\sigma_{n}^{*}\left\{\exp \left(\frac{E F}{R T}\right)-1\right\}+\sigma_{p}^{*}\left\{1-\exp \left(-\frac{E F}{R T}\right)\right\}\right]
$$

The above equation indicates that current is a function of electron and hole conductivities at the specific partial pressure of oxygen. Therefore, voltage measurement at the steady-state under a specific partial pressure of oxygen at applied constant current can provide data sets as shown in Fig. 8.

Based on these data after proper fitting, we calculated the partial conductivity of electron and hole in the 8YSZ system as:

$$
\begin{aligned}
& \sigma_{n}=2.42 \pm 0.23 \times 10^{7} \exp (-3.99 \pm 0.17 e V / k T)\left(p O_{2}\right)^{-1 / 4} \\
& \sigma_{p}=1.30 \pm 0.15 \times 10^{2} \exp (-1.70 \pm 0.06 e V / k T)\left(p O_{2}\right)^{1 / 4}
\end{aligned}
$$

And, by using the calculated partial electric conductivities and the total conductivity by the 4-probe DC measurement, we calculated the ionic conductivity as:

$$
\sigma_{i o n}=2.24 \pm 0.10 \times 10^{2} \exp (-0.80 \pm 0.01 \mathrm{eV} / \mathrm{kT})
$$

Under the normal partial pressure of oxygen range (Air$\mathrm{H}_{2}$ ) in SOFC, 8YSZ showed superior ionic conductivity, and

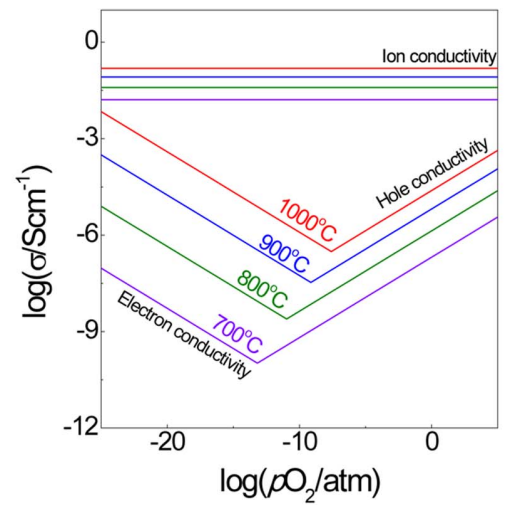




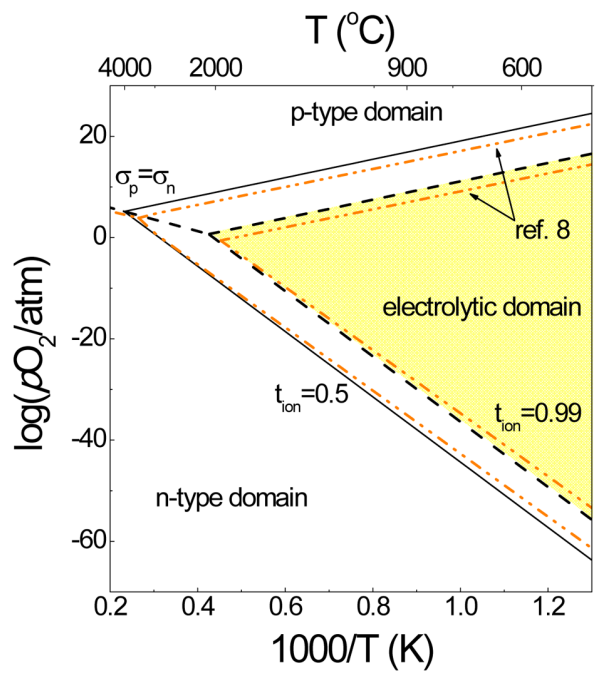

Fig. 9. Calculated boundaries in electrolytic domain of the 8YSZ.

was in good agreement with reported ionic conductivity of $0.79 \mathrm{eV}^{8}$ within the error range. From these results, upper and lower limits of oxygen partial pressure $\left(\mathrm{P}_{\mathrm{p}}\right.$ and $\left.\mathrm{P}_{\mathrm{n}}\right)$, and oxygen partial pressure $\left(\mathrm{P}_{\mathrm{pn}}\right)$ where concentrations of electron and hole become equal are calculated in the electrolyte domain, where the transport number of oxygen ion is above 0.5 :

$$
\begin{aligned}
& P_{p}=\left(\frac{A_{i}}{A_{p}}\right)^{4} \exp \left[-\frac{4\left(E_{i}-E_{p}\right)}{k T}\right] \\
& P_{n}=\left(\frac{A_{i}}{A_{n}}\right)^{-4} \exp \left[\frac{4\left(E_{i}-E_{n}\right)}{k T}\right]
\end{aligned}
$$

As shown in Fig. 9, the electrolytic domain covers wide ranges of both partial pressure of oxygen and temperature. For example, electrolytic domain at $t_{\text {ion }} \geq 0.99$ at $700^{\circ} \mathrm{C}$ extends oxygen partial pressure range across $10^{11}-10^{-38} \mathrm{~atm}$., while it is $4.5 \mathrm{~atm}$. at $2000^{\circ} \mathrm{C}$, which is in good agreement with the reported values by Park et al. ${ }^{8)}$

\section{Conclusions}

We prepared an electrolyte specimen for SOFC to measure its partial conductivities using a commercial 8YSZ powder. For the measurements with better accuracy, we designed and prepared an improved version of Hebb-Wagner polarization cell. Based on the defect reaction model, which states that conductivities by electron and hole are proportional to the partial pressure of oxygen to the powers of $-1 / 4$ and $1 / 4$, respectively, we performed Hebb-Wagner polarization experiments in the temperature range of 700 $1000^{\circ} \mathrm{C}$. We confirmed that the partial conductivities for electron and hole are negligibly low $\left(1.65 \times 10^{-5}\right.$ and $5.71 \times$ $10^{-9}$, respectively) at $1000^{\circ} \mathrm{C}$ in air, compared to $1.52 \times 10^{-1}$ for ionic conductivity. We observed the same trend in the other temperature range, which showed ionic transport numbers higher than 0.99. We thus demonstrated that the prepared $8 \mathrm{YSZ}$ is an appropriate electrolyte for SOFC with excellent oxygen ion conductivity under the given conditions of this study.

\section{Acknowledgments}

This work was supported by the Industrial critical technology development program (Grant no. 10049161), "Technological development of a process for manufacturing highpurity zirconia and the applications/products using zircon sand feed" funded by the Ministry of Trade, Industry \& Energy (MI, Korea).

\section{REFERENCES}

1. C. Song, L. Zhang, J. Zhang, D. P. Wilkinson, and R. Baker, "Temperature Dependence of Oxygen Reduction Catalyzed by Cobalt Fluorophthalocyanine Adsorbed on a Graphite Electrode," Fuel Cells, 7 9-15 (2007).

2. R. Noyes, Energy Technology Review; Vol. 1, pp. 1-32, Noyes Data Corporation, New Jersey, 1977.

3. S. Sengodan, S. Choi, A. Jun, T. H. Shin, Y. W. Ju, H. Y. Jeong, J. Y. Shin, J. T. S. Irvine, and G. T. Kim, "Layered Oxygen-deficient Double Perovskite as an Efficient and Stable Anode for Direct Hydrocarbon Solid Oxide Fuel Cells," Nat. Mater., 14 205-9 (2015).

4. A. Trovarelli, "Catalytic Properties of Ceria and $\mathrm{CeO}_{2}$-Containing Materials," Catal. Rev. Sci. Eng., 38 [4] 439-520 (1996).

5. B. C. H. Steel, "Appraisal of $\mathrm{Ce}_{1-\mathrm{y}} \mathrm{Gd}_{\mathrm{y}} \mathrm{O}_{2-\mathrm{y} / 2}$ Electrolytes for IT-SOFC Operation at $500^{\circ} \mathrm{C}$," Solid State Ionics, 129 95110 (2000).

6. M. H. Hebb, "Electrical Conductivity of Silver Sulfide," J. Chem. Phys., 20 185-90 (1952).

7. J. H. Kim and H. I. Yoo, "Partial Electronic Conductivity and Electrolytic Domain of $\mathrm{La}_{0.9} \mathrm{Sr}_{0.1} \mathrm{Ga}_{0.8} \mathrm{Mg}_{0.2} \mathrm{O}_{3-8}$," Solid State Ionics, 140 105-13 (2001).

8. J. H. Park and R. N. Blumenthal, "Electronic Transport in 8 Mole Percent $\mathrm{Y}_{2} \mathrm{O}_{3}-\mathrm{ZrO}_{2}$," J. Electrochem. Soc., 136 [10] 2867-76 (1989). 